\title{
Severe Paediatric Multisystem Inflammatory Syndrome (PIMS) in a 23 months old baby post COVID-19 effectively managed with IVIG and pulse Steroid: Case report
}

Dr Yogesh Kumar Gupta ( $\nabla$ dygupta83@gmail.com )

Dept Of Paediatrics and Paediatric Critical care,Fortis Hospitals, BG Road, Bangalore 76 https://orcid.org/0000-0001-5437-8007

\section{Dr Sowmya Shenoy}

Fortis Hospitals, BG Road, Bangalore 76

Dr Kuldip G Paike

Dept of Paediatrics and Paediatric Critical care,Fortis Hospitals, BG Road, Bangalore 76

Dr Mrigendra Nath Tudu

Dept of Paediatrics and Paediatric Critical care,Fortis Hospitals, BG Road, Bangalore 76

\section{Case Report}

Keywords: Coronavirus disease, Covid-19, Hyperinflammatory syndrome in children, immuno-modulatory drugs

Posted Date: September 2nd, 2020

DOl: https://doi.org/10.21203/rs.3.rs-68719/v1

License: (c) (1) This work is licensed under a Creative Commons Attribution 4.0 International License. Read Full License 


\section{Abstract}

Coronavirus disease (COVID-19) in children largely causes mild disease. Some of these recovered children may present with delayed manifestations described as Hyperinflammatory syndrome resembling closely with Kawasaki disease (KD) or Toxic Shock Syndrome (TSS). Timely identification, supportive treatment and treatment with immunomodulatory drugs make a significant difference in the outcome.

\section{Background}

Majority of children are without symptoms or mild symptoms from Covid-19 infection [1]. It is known to be severe in children with co-morbidities ${ }^{[2]}$. There are clusters of children and adolescents who have multisystem inflammatory condition with symptoms similar to Kawasaki Disease (KD) or Toxic Shock

Syndrome (TSS) ${ }^{[3]}$. It is known in cities which have reached peak of case incidences or nearing its peak ${ }^{[4]}$ .There is an increasing necessity for similar case reporting for understanding its risk factors, causality and describe treatment interventions. Here, we report a case of Severe Paediatric Multisystem Inflammatory Syndrome in a previously normal child.

\section{Case Presentation}

A 23 month old male baby presented to us with h/o fever for 3 days, loose stools of 2 days duration with oligouria since 24 hours. Baby was resuscitated with ringers lactate bolus in view of prolonged capillary refilling time. On examination, he had non-purulent conjunctivitis, periorbital puffiness with edema of dorsum of hand and feet with non-tender hepatomegaly. Following resuscitation, first dose of IV antibiotics were given with a suspicion of sepsis after sending relevant workup. RT-PCR Sars-Cov2 was negative at admission. Initial investigations revealed anaemia ( $\mathrm{Hb}-9.6 \mathrm{gm} \%)$, Leucocytosis with significant lymphopenia (TC-11960/cumm, N= 85\%, L= 13\%, Absolute Lymphocyte Count 1.59 thousand/microL), mild thrombocytopenia (90 thou/micL) ,Hyponatremia $(127 \mathrm{mmol} / \mathrm{l})$ with markedly elevated inflammatory markers [high CRP $-148.5 \mathrm{mg} / \mathrm{L}$, elevated ESR $-60 \mathrm{~mm}$ at one hour, procalcitonin- $211.7 \mathrm{ng} / \mathrm{mL}$, Ferritin$818.2 \mathrm{ng} / \mathrm{mL}$, IL-6- $118.6 \mathrm{pg} / \mathrm{mL}$ ). Child also had pre-renal AKI (creatinine-1.73 mg/dL, urea- $128 \mathrm{mg} / \mathrm{dL}$, BUN-60 mg/dL) with mildly elevated liver enzymes (AST/ALT - 75/79 IU, INR-1.12). Within 6 hours of admission child developed hypotension requiring initiation of inotropic support (Dopamine followed by Nor-Epinephrine). With a possibility of Hyper inflammatory syndrome with Multi-organ involvement (based on clinical features and presentation) NT-Pro-BNP sent which was markedly elevated $(40684 \mathrm{pg} / \mathrm{ml})$ suggesting severe cardiac involvement. Child was given IVlg (2gm per $\mathrm{Kg})$ along with pulse Methyl prednisolone (10 mg per $\mathrm{kg}$ for three days). Child required High flow nasal cannula support for respiratory distress. D-Dimer was elevated $(4.4 \mathrm{mcg} / \mathrm{mL})$ and prophylactic subcutaneous Low molecular weight heparin was started. Bedside ECHO showed mild biventricular dysfunction on inotropic support with normal coronaries. SARS-COV2-IgG came positive (5.43 index). Aspirin (50mg) was started on day 3 of hospitalization once thrombocytopenia improved. Parents were tested positive for IgG covid-19 antibodies. 
With above management, baby started showing improvement with decreasing respiratory distress and decreasing requirement of inotropic support. Child was taken off inotropic support in next 48 hours and started on diuretics for preload reduction. Oral feeds were established on day 3 of admission which baby tolerated well. Baby was noted to have sinus Brady-arrhythmias (normal PR interval with normal hemodynamics and normal ejection fraction) hence no active intervention was done. Antibiotics were deescalated (as per Infectious disease specialist) once cultures were sterile. Repeat inflammatory markers showed decreasing ESR, Procalcitonin, IL 6 levels and normalizing Absolute lymphocyte count.

\section{Discussion}

Paediatric Multisystem Inflammatory Syndrome in Children and adolescents post Covid-19 is a relatively new clinical entity described in literature. Its symptoms are somewhat similar to the symptoms in Kawasaki Disease. Cases are being reported from cities like Mumbai, Chennai and Delhi, which tend to occur after weeks of primary Covid infection ${ }^{5,6}$. This particular child is one of the few first cases reported from Karnataka which is nearing its peak of Covid- 19 cases. Case definition is suggested by WHO: fever

for $\geq 3$ days and rash, non-purulent conjunctivitis, shock, coagulopathy, myocardial dysfunction, gastrointestinal involvement and elevated inflammatory markers with no evidence of bacterial sepsis with evidence of COVID-19(RT PCR, Antigen or Serology positive). Most common age group described in literature is beyond 5 years of age and in adolescents, whereas it was a young baby ( 23 months) in our case report. NT Pro-BNP correlates well with extent of cardiac involvement. In our case it was among the highest reported so far in literature. Various immunomodulators have been tried in its treatment including IVIG, Steroids and Interleukin antagonists.

\section{Conclusion}

Timely identification of hyperinflammatory syndrome with appropriate management of shock and prompt administration of IVIG with pulse dose of methyl prednisolone can effectively stop Cytokine storm leading to favorable outcome. Despite IL 6 being high ,there are fair chances these children may not need biological agents if responsive to initial immunomodulatory treatment. Though further studies are needed to study the cardiac outcome in these children.

\section{Declarations}

Competing interests: The Authors declare "no competing interest"

Consent: I declare that parents of the child in this particular case report have given their consent to participate and publish the data and facts mentioned in this report.

\section{References}


1. Royal College of Paediatrics and Child Health. Guidance - Paediatric multisystem inflammatory syndrome temporally associated with COVID-19 (https://www.rcpch.ac.uk/resources/guidancepaediatric-multisystem-inflammatory-syndrome-temporally-associated-covid-19-pims? _sm_byp=iVVV13NFfQv0T10j)

2. Infection and transmission among children/CDC https://www.cdc.gov/coronavirus/2019ncov/hcp/pediatric-hcp.html

3. WHO guidance on multisystem inflammatory syndrome in children and adolescents with covid-19 (https://www.who.int/news-room/commentaries/detail/multisystem-inflammatory-syndrome-inchildren-and-adolescents-with-covid-19).

4. Journal of Pediatric Infectious diseases society (https://www.researchgate.net/journal/20487207_Journal_of_Pediatric_Infectious_Diseases_Society)

5. Epidemiological and Clinical Profile of Pediatric Inflammatory Multisystem Syndrome - Temporally Associated with SARS-CoV-2 (PIMS-TS) in Indian Children. K Dhanalakshmi , Aishwarya Venkataraman , S Balasubramanian, Manoj Madhusudan,Indian paediatrics, Aug 6, 2020

6. Rowley AH. Multisystem inflammatory syndrome in children and Kawasaki disease: Two different illnesses with overlapping clinical features [published online ahead of print, 2020 Jun 22]. J Pediatr. 2020;S0022-3476(20)30760-5.

\section{Supplementary Files}

This is a list of supplementary files associated with this preprint. Click to download.

- Reporttable14.docx 